\title{
An Interpretive Descriptive Approach to Understanding Osteoporosis Management from the Perspective of People with Osteoporosis
}

\section{Christina Ziebart ( $\nabla$ cziebart@uwo.ca )}

Western: Western University https://orcid.org/0000-0002-3667-4133

Joy MacDermid

Western University

\section{Rochelle Furtado}

Western: Western University

Mike Szekeres

St Joseph's Health Centre

Nina Suh

St Joseph's Health Centre

Aliya Khan

McMaster University

\section{Research Article}

Keywords: Osteoporosis; qualitative; health management; exercise, falls

Posted Date: March 2nd, 2022

DOI: https://doi.org/10.21203/rs.3.rs-800953/v1

License: (c) (P) This work is licensed under a Creative Commons Attribution 4.0 International License. Read Full License 


\section{Abstract}

Background: Adherence to both non-pharmacological and pharmacological fracture prevention interventions is often low in people with osteoporosis. Understanding how patients acquire information about osteoporosis management is important for understanding both the initial decision-making and ongoing adherence. This study explored the narrative of people living with osteoporosis and their personal experience getting information about their osteoporosis management.

Methods: An interpretive descriptive method was used for this qualitative study. In-depth interviews were conducted with 13 Canadian participants (age range 51-90) who knew that they had osteoporosis or osteopenia. Participants were asked to participate in one-on-one interviews to address the type of health professionals providing osteoporosis management advice focusing specifically on advice received about exercise, nutrition, and falls prevention. Interviews were transcribed verbatim and coded sentence-by-sentence.

Results: People with osteoporosis rely on physicians for advice related to pharmacological treatment needs, and other health professionals for non-pharmacological needs such as exercise advice, nutrition advice, and falls prevention advice. People value non-professionals, such as family members and close friends, who may or may not have osteoporosis, to discuss or corroborate health professional advice, or to validate their belief system.

Conclusion: Training patients to more effectively engage in conversations with their healthcare providers may be a strategy to improve the quality of communication and its translation into adherence to best practices in managing osteoporosis.

\section{Background}

Osteoporosis is characterized by low bone mass and a decrease in bone mineral density. Osteoporosis affects both 1 in 3 women and 1 in 5 men and is associated with a large economic burden[1]. Clinical recommendations for the management of osteoporosis include assessing men and women over the age of 50, individuals that have sustained a fragility fracture over the age of 50 , or those with high fracture risk[2]. Evaluation for fracture risk consists of measuring patient height yearly, assessing their fall history and measuring bone mineral density[2]. Other biochemical and lifestyle factors may also be evaluated to determine the 10-year fracture risk[2]. The 10year fracture risk categorizes participants into a low, moderate, or high risk of fracture. Typical management strategies rely on the categorization to determine the best course of action.

For people categorized as low or moderate risk, a non-pharmacological strategy is often recommended. Participants will be encouraged to increase their dietary calcium, ensure they are obtaining sufficient vitamin D (often requires supplementation), and finally ensure they are sufficiently loading their bones through exercise. Osteoporosis exercise recommendations suggest that people with osteoporosis engage in a strength and balance training program, practice spine sparing strategies and posture re-training[3]. It is recommended that people with osteoporosis seek out a health care professional (such as a physical therapist or kinesiologist) that is trained in providing exercise recommendations to people with osteoporosis to support appropriately targeted and implemented exercise. Pharmacologic intervention is also advised for people in the moderate fracture risk who experience bone loss or have diseases associated with bone loss or medications associated with bone loss or in the presence of falls as falls are an independent risk factor for fracture independent of bone mineral density (BMD)[4]. 
For people categorized in a high-risk category, in addition to the non-pharmacological management, it is recommended that participants engage in a pharmacological management strategy. There are several pharmacological treatments available, which are prescribed by the physician and determined on an individual basis. Based on the osteoporosis recommendations, thorough management of osteoporosis requires a collaborative effort from both physicians and non-healthcare providers.

To date, no study has evaluated the perception of people with osteoporosis in being able to navigate the healthcare system to manage their osteoporosis. Engaging in osteoporotic health care recommendations demonstrate a challenge for people with osteoporosis. This is evident as adherence with pharmacological treatment to prevent fractures is reportedly low[5-7]. Similarly, the non-pharmacological recommendations such as, behaviour change to engage in osteoporosis exercise, have also been reported to be challenging[7]. People with osteoporosis feel that they are not equipped with enough knowledge to engage in an exercise program independently[8], but are also not always able to access health care professionals capable of guiding their exercise routine $[9,10]$. Access to the appropriate type of health professional may relate to the supply of available health providers, their geographic proximity, and the funding models that determine who pays for the services. Although clinical practice guidelines have been published for health care providers[2, 3], there continues to be gaps in the patient's perception on how to manage their osteoporosis.

Therefore, the purpose of this study is to understand the patient's perception of managing their declining bone mineral density. This study will explore the narrative of people living with osteoporosis or osteopenia and their personal experience obtaining information about their osteoporosis management. The aim of this study was to explore the experience of managing osteoporosis from the perspective of people living with osteoporosis or osteopenia.

\section{Methods}

An interpretive descriptive methodology was used for this qualitative study[11, 12]. Interpretive description is aligned with constructivist and naturalistic style of inquiry $[12,13]$. This methodology is commonly used in a clinical context of applied health disciplines[12,13]. The strength of interpretive descriptive methodologies is that there is a coherent logic and structure designed towards the generation of practice-relevant findings[12, 13]. However, there are also challenges in the degree of interpretation and that this is a lesser-known methodology[12, 13]. Generally, the methods for interpretive descriptive methodologies are similar to that of traditional qualitative methods but acknowledges that the researcher is involved in the study and may not be completely impartial[13].

\section{Participants and procedures}

In-depth interviews were conducted with 13 Canadian participants (age range 51-90) that knew they had osteoporosis or osteopenia, as told by their physician, taking place between February to April of 2019. Participants were asked to participate in a one-on-one interview (Interview guide presented in Appendix 1). The interview guide was piloted with a first participants, but no major changes were made and therefore the data was included in the overall sample. Participants were encouraged to speak freely and openly about their experience with osteoporosis. The semi-structured interview guide was structured to prompt discussion around which medical professionals the participants have seen for their osteoporosis management, the advice they received about managing their osteoporosis, and a specific focus on whether they had received advice about exercise, 
nutrition, and falls prevention for osteoporosis. Goals and knowledge gaps related to osteoporosis management were explored to further understand how their osteoporosis care could be modified to better suit the requirements of living with osteoporosis.

Participants were selected from a medical clinic in Ontario, Canada. Eligible participants were selected based on having osteoporosis or osteopenia, being able to speak and understand English, and capable of providing informed consent to participate. Patients with secondary osteoporosis were excluded from this study. Participants were approached in the clinic and were provided with the letter of information and consent. Interested participants reached back out to the lead student researcher to set up an interview day and time. Participants had the opportunity to ask any questions about the letter of information before signing consent. The interview took place in a location of the participant's preference, often a Starbucks. Participants were reminded that their conversation was taking place in a public location and to be mindful of that when answering questions. Participants signed the informed consent prior to being interviewed. The interviews were audio-recorded on a digital recorder. To achieve purposeful sampling, recruitment consisted of women in the earlier stages post-menopause, older women, and men. The sample provides both depth and different dimensions of emerging themes. The data were analyzed throughout the data collection period to allow for constant comparison and an indication of when theoretical saturation was reached. Constant comparison was used to assess the quality of the questions being asked, verifying that participants understood the questions, and allowing the evolution of questions. For example, it emerged that participants valued non-healthcare providers advice for their osteoporosis, so future iterations of the interview guide explored that theme more thoroughly. Data collection was ceased when theoretical saturation was reached, which is when no new information was provided in the last 2 interviews.

This study was approved by Western University's Research Ethics Board and Lawson Health Research Board.

\section{Analysis}

Data analysis occurred manually and simultaneously with the data collection[11]. The interviews were stopped once theoretical saturation was reached. A single researcher (CZ) transcribed all the interviews verbatim. Two researchers (CZ and RF) analyzed all transcripts. Data was coded sentence-by-sentence to identify emerging themes[11]. Themes were organized into categories, which were dividing and rebuilt into concepts[11]. The themes, categories and concepts were constructed and integrated into an explanatory theory[11].

\section{Open coding}

The first step of the coding process was through open coding [11]. The codes were established sentence-bysentence, for the whole text of every interview. The codes were written in the document of the transcription and compared to reveal potentially significant phenomenon and recurring themes. A codebook was created to document the codes during this process. The codebook was modified with each new interview adding new emerging codes. Recurring themes were determined as concepts that were similar. These common themes were then grouped into higher-level abstraction for the development of emerging subthemes.

\section{Axial coding}

Axial coding was used to define subthemes and link them with other related codes[11]. Discussion, constant comparison and memo-writing of the subthemes and codes that emerged when analyzing the data were documented to being categorizing the codes into subthemes until it seemed that no further subthemes could be 
established, and theoretical saturation was reached[11]. Each subtheme was assessed to ensure that it influenced the phenomenon explored.

\section{Selective coding}

In this final part of the analysis, we focused on compiling themes that reflected knowledge sources for how people with osteoporosis managed their osteoporosis[11]. The categories that emerged were organized and compared to assess that the characteristics were able to explain the phenomenon studied. Differences of themes across different types of health professionals emerged (physician, non-physician health professional and nonhealthcare providers) and coding was completed under these umbrella categories[11].

\section{Results}

A total of 13 participants were interviewed. The average length of the interview was 45 minutes, with the shortest interview at 34 minutes and the longest interview at one hour and 15 minutes. The mean age of 66 , mean age at menopause was 46, and mean number of fractures was two. A total of 12 females and one male was included, three participants have osteopenia and seven have osteoporosis, and two participants self-reported that they did not know (however the study physician did confirm they had osteoporosis). The fracture risk included participants in low, moderate and high risk of fracture (Table 1). The themes in each focus on the type of information which people living with osteoporosis rely on from their physician, other healthcare professionals, and non-healthcare providers. Specifically, participants rely on the physicians for disease information and advice related to pharmacological treatment needs, whereas they rely more on other health professionals for non-pharmacological needs such as exercise advice, nutrition advice and falls prevention advice, and non- healthcare providers for advice corroborating health professional advice, or to validate their belief system. A total of 10 themes and 11 subthemes were identified. The themes are summarized in Fig. 1. 
Table 1

Participant demographics and response to demographic survey data

\begin{tabular}{|c|c|c|c|c|c|c|c|c|}
\hline ID & Age & $\begin{array}{l}\text { Age at } \\
\text { menopause }\end{array}$ & Sex & $\begin{array}{l}\text { Primary } \\
\text { dwelling }\end{array}$ & $\begin{array}{l}\text { Osteoporotic } \\
\text { fracture }\end{array}$ & Diagnosis & $\begin{array}{l}\text { Fracture } \\
\text { Risk }\end{array}$ & $\begin{array}{l}\text { Osteoporosis } \\
\text { Medication }\end{array}$ \\
\hline 01 & 69 & 52 & Female & City & $\begin{array}{l}\text { 2: both left } \\
\text { wrist }\end{array}$ & Osteoporosis & High & Yes \\
\hline 02 & 62 & Early 50 s & Female & $\begin{array}{l}\text { Rural } \\
\text { area }\end{array}$ & None & Osteoporosis & Moderate & No \\
\hline 03 & 64 & 55 & Female & City & None & Osteopenia & Low & Yes \\
\hline 04 & 70 & 55 & Female & City & None & Osteoporosis & High & No \\
\hline 05 & 51 & 45 & Female & City & $\begin{array}{l}\text { 2: hand and } \\
\text { wrist }\end{array}$ & Osteoporosis & High & Yes \\
\hline 06 & 90 & $\begin{array}{l}\text { Not } \\
\text { applicable }\end{array}$ & Male & City & $\begin{array}{l}\text { 2: right and } \\
\text { left arm }\end{array}$ & Osteoporosis & $\begin{array}{l}\text { Do not } \\
\text { know }\end{array}$ & Yes \\
\hline 07 & 88 & Early 50 s & Female & City & None & Osteopenia & $\begin{array}{l}\text { Do not } \\
\text { know }\end{array}$ & Yes \\
\hline 08 & 57 & 36 & Female & City & None & Osteoporosis & Moderate & No \\
\hline 09 & 60 & 54 & Female & City & 1 : wrist & Osteoporosis & $\begin{array}{l}\text { Do not } \\
\text { know }\end{array}$ & No \\
\hline 10 & 66 & 50 & Female & City & 1 : wrist & Osteoporosis & Moderate & Yes \\
\hline 11 & 62 & 30 & Female & City & None & Osteopenia & High & No \\
\hline 12 & 61 & 50 & Female & City & None & Do not know & $\begin{array}{l}\text { Do not } \\
\text { know }\end{array}$ & No \\
\hline 13 & 59 & 30 & Female & City & None & Osteoporosis & $\begin{array}{l}\text { Do not } \\
\text { know }\end{array}$ & No \\
\hline
\end{tabular}

Table 2: Knowledge gaps and comments where the knowledge was incorrect 


\section{Knowing how to put together an exercise program}

2. Improve knowledge on intensity of exercise

3. Improve knowledge that walking isn't enough

4. Lacking specific osteoporosis knowledge

5. Learning about advice that is appropriate for osteoporosis

6. Recognizing the severity of osteoporosis

7. Lack of knowledge on test results

8. Lack of knowledge on how weight loss diets affect osteoporosis

9. Thinking osteoporosis will increase fall risk

10. Exercise program will increase bone mineral density

11. Learning better exercise form

12. Only seeking information when bone mineral density goes down

13. Knowing the medication side effects

14. Learning about how much calcium is in the foods they eat

15. Exercise contraindications

16. Which exercises are safe for osteoporosis

17. How to personalize the exercise program

18. Training and depth of knowledge related to osteoporosis for personal trainers and physiotherapists

19. Fracture risk and bone mineral density, how they relate and how they differ

20. Quality of exercise versus quantity of exercise

21. How to get sufficient nutrients from alternative diets

22. Understanding the bioavailability and absorption of calcium

Physicians

Communicating with the physician

A common theme was that people living with osteoporosis relied on their physicians for information about the disease. Although the physician who ultimately diagnosed their osteoporosis or set them on the pathway to manage it varied, it was clear that communication with a physician was an important part of the initial management of osteoporosis. Many participants sought the help of an osteoporosis specialist, but also relied on the advice from their family doctor. In one instance, it was an emergency room doctor that initiated the trajectory towards a diagnosis of osteoporosis, after the participant fractured their wrist. In some instances, the participants described their osteoporosis as being not very severe as they were seen by a specialist and then returned back to their family doctor as the primary point of contact for managing their osteoporosis. In some cases, establishing 
that therapeutic alliance between a physician who helped to them manage their osteoporosis was not a simple process as illustrated by one respondent who said that:

"the original GP I spent a lot of time not seeing her, because I really did not feel comfortable with her. It was really my way or the highway, and she was tell tell tell and never a listen listen listen. So, I finally did find a GP... And I am following her, and I quite enjoy her. So she is starting to build that relationship now, that I can talk through those things and she has a nurse practitioner there too." -- Female, age 69.

A positive relationship with the physician seems to be an important aspect of managing osteoporosis as a disease.

Responsibility of explaining osteoporosis as a disease

\section{Understanding disease severity and how it impacts treatment}

Physician care is usually the first point of contact for people with osteoporosis. Participants frequently mentioned that they saw a physician in the process of getting diagnosed with osteoporosis. Ensuring that the physician is clear about the delivery of the osteoporosis diagnosis seemed to be a critical factor in how participants understood the severity of the disease and managed their osteoporosis accordingly. One participant said that her trajectory for getting an osteoporosis diagnosis began after fracturing her wrist, where her family doctor:

"recommended [her] to the [hospital] because [she] had two falls and broken [her] wrist. And they were falls, that from [her] perspective, yeah you would have broken your wrist. So [she] wouldn't have otherwise thought about it, but they said you know what you have to go and get tested and do the bone density and get tested here. So that's where it came from"-- Female, age 69.

However, another participant felt that she was lacking information related to her osteoporosis management from her family doctor because "like you know like with the family doctor you're in and out. She's good at following up on my bone density scans and medication if it's needed. I never feel like 100\% that you know l'm taking the right vitamins and stuff like that"- female, age 59; reiterating that "I pretty much don't even have enough knowledge to know what I'm lacking'- Female, age 59. There is a sense that the person with osteoporosis is not managing their osteoporosis fully because they do not appreciate the severity of the disease, where participants mentioned that they cannot feel the disease and avoid taking medication or continue doing activities that may increase fracture risk.

\section{Physicians can be the gateway to other health professionals}

The physician often relays information about the need for, or role of, other health professionals to help their patient access help with non-pharmacological management. For one participant, her progression from osteopenia to osteoporosis motivated her physician to recommend involving other health professionals:

"the last time I was, when I got the diagnosis having moved from osteopenia to osteoporosis, [the doctor] did not mention exercise or programs of exercise... [but] when [she] came back end of January, [the doctor] mentioned [other health professionals]"- Female, age 57,

Finding out how other health professionals can complement pharmacological management of osteoporosis was a pivotal moment for this very active participant to learn how to exercise and move safely with osteoporosis. 


\section{Understanding the role of pharmacological care in osteoporosis Explaining and managing medication side effects}

The side effects of medications and the extent to which they understood those potential side effects before starting a new osteoporosis medication were a concern for some of the participants. One participant in particular explained that she was experiencing some pain in her jaw which she attributed to her osteoporosis medication injection:

"I expressed concern to her about my jaw. I said it really is troublesome. Even when I'm relaxed watching television [I was in pain]. She said oh no [osteoporosis drug] does not have side effects in the jaw. Well it got worse. The worst thing with having an injection, I might just inject this into the conversation, is that if you're taking by a shot you can't stop taking it. If you notice any side effects. Not like taking a pill or something more regularly once a month. When you have the [osteoporosis drug], you've had it. So now l've had it twice in my system. And so I had to ride out the next 6 months, and then I just didn't go for it 6 months later." -Female, age 70.

This patient's concern reflects critical piece of information that might affect decision making since unlike medications which you can stop taking and expect side subside relatively quickly, side effects from injections may take months to dissipate. Osteoporosis medication injections may be administered twice a year and patients felt like their only option was to not repeat the intervention if they experienced an unacceptable side effect. Some participants expressed concerns that the side effects of medications may not be fully understood by physicians. One participant had a daughter that was on long-term medications that resulted in complications

"She took a lot of cortisone over the years, which also diminishes your bones. Then she developed osteoporosis and her doctor put her on [osteoporosis drug] and put her on for 10-15 years, and she should have been on it for 3' -Female, age 88.

Her daughter's experience also affected her decision making around her own osteoporosis, since her negative perceptions about her daughter's experience made her resistant to beginning medication, and instead, she chose to manage her osteoporosis non-pharmacologically.

\section{Lack of shared decision-making or understanding of the benefits and limitations of the medication}

Decision making around medication was a recurring theme for the participants, with most participants reporting feelings that these concerns had not be adequately addressed. The lack of understanding indicated by participants suggest that either there was limited explanation and/or the explanations were not being understood by the participants. One participant expressed this frustration:

"I wanted to know more about [osteoporosis drug] for instance. How long you should be on it, because of [the participant's daughter'] experience with [osteoporosis drug], and she gave it to me, with one pill and that was it. I wouldn't take it. I don't know why I took [osteoporosis drug], I guess I just gave in. No, I find she doesn't take the time to listen to the question you might have. She has too many people lined up one after the other in the office."Female, age 88. 
Other participants have expressed their concern with going off medication because they would like to try nonpharmaceutical options. Particularly, participants often wanted to know what the rate of their bone loss would be and whether it was worth pursuing a course of non-pharmaceutical treatment before having to make the decision about implementing medication. Some experienced a lack of physician support for this approach. One participant illustrated a thoughtful decision-making around benefits and limitations of medication, and was hesitant to start medication without such a trial but felt that her physician was pushing her towards taking medication:

"yup so I had an appointment set up for a couple months back for the nurse to give me an IV, and I called and cancelled that and I would like to speak with the doctor at that time. But it wasn't possible to do that. So I waited another couple of months and went in to see [the doctor] and I told her. And she seemed relatively professionally angry with me. Thinking perhaps I am being foolish. [Her] arguments are very reasonable. There have been clinical trials. You're not a guinea pig. People have been doing this for years. The side effects are listed it's because a very small percentage of the population has this difficult. And I said yes, yes, but I don't want to take the drug. I want to have another opportunity to do something to try to decrease. And I asked what is the rate of bone loss and how much danger am I in? And she said it, she looked at the information and she showed me the information and I said I don't understand that, and she explained. And the rate of bone loss is rather regular with me. So, if I lose another $0.02 \%$ between now and when I see you next, would that be significant? And she said she didn't think that would be terribly significant. I said that I would like to wait and see how the exercise and diet changes. Whether they have any impact. And she said they won't. She said it's good to exercise and have good nutrition, but she said this is going to happen, you are on a pathway and you're going to end up with bone loss. And I said okay. And at that point I might take the drug. An it sounds like it's really good to slow the progress of the disease." - Female, age 57.

Beyond the hesitation of initiating medication, participants expressed concern with having to travel with medication and not knowing if it would travel safely due to the storage recommendations. Some osteoporosis drugs require refrigeration, and one participant wasn't sure if she would be able to comply with that while on a plane.

Unexpectedly, few participants mentioned any financial restrictions with going on medication, likely due to having external health coverage, or sufficient financial means to cover the drug costs. Rather, it seemed the interruption in lifestyle and hesitation to take any medication limited medication use in these participants.

\section{Providing advice to maintain or modify exercise routines}

One of the biggest concerns for the younger participants after getting diagnosed with osteoporosis was to be able to return to their pre-osteoporosis exercise routine. The participant found that the advice provided to her from her physician, while conservative, might not have considered her own fitness levels. She said that the physician's "advice regarding exercise was very troublesome to me" because the physician advised her "stop cycling which is a very big part of [her] life. [The doctor] said [she] can walk. And when [she] said what about yoga [the doctor] said there are all sorts of twists you can't do."; the way in which this participant was told to manage her osteoporosis was very hard for the participant since she did not fully understand the rationale for these recommendations.

\section{Other Health Professionals}


Many of the participants interviewed sought advice for their osteoporosis beyond their physician. Other health professionals that participants reported seeing included physical therapists, massage therapists, naturopath, dietitian, acupuncture, osteopath and kinesiologists.

\section{Understanding the role of non-pharmacological care in osteoporosis}

\section{Understanding when to see certain health care professionals}

Generally, from the interviews, participants were unsure about which health care professionals to see, what to see them for, and at what stage of their osteoporosis required which healthcare professional versus their physician. One participant mentioned the information related to her osteoporosis management she:

"certainly, didn't have it handed to [her]. It was something [she] needed to pursue on [her] own and research. [The doctor] was helpful in saying here the document you can read about and here's the website you can go to. But the onus was really on [herself] to take advantage of that. There really wasn't anyone guiding [her]."-Female, age 51.

This participant found that she had to take on the responsibility of finding a physiotherapist or kinesiologist knowledgeable in osteoporosis management to help her with her exercise. However, once the participants were in contact with another health professional, the experience seemed positive, saying that working with "the kinesiologist, excellent"- Female, age 51; "I did find the physio very good in terms of the exercises... And the exercises, how she talked about the importance of the core and core exercises. And since l've been following those core exercises I've noticed a huge improvement'- Female age 59. Despite one participant not feeling supported in finding another health professional, many of the participants were referred to other health professionals from their physician or sought their physician's advice on what other health professionals to see.

\section{Understanding the benefits and limitations of exercise, nutrition, and falls programs}

For participants that are already active, providing an exercise routine that suits their lifestyle was an important component of their osteoporosis management. One very active participant considers her active lifestyle part of her identity but understood that the exercises she was doing may need to be modified for her osteoporosis. Recognizing that she:

"wanted to know what safe exercises were. Because [she's] not prepared to give up exercising. And [she doesn't] think it's good for [her] diagnosis to just start walking and that's it. And [she goes] to the gym and [she] workout and [she] use weights. And [she] want[s] to be able to do that safely. [She wants] to be able to strengthen a muscle without breaking a bone while [she's] doing it. So [she] wanted to ask the physiotherapist how to do that... So to be able to have [a kinesiologist] come to [her] gym and have [the kinesiologist] look at what [she's] doing and say 'no stop that, but here's an alternative' is just fantastic. And [she's] really excited about that." - Female, age 57

Understanding that there are alternatives to exercise and there are safe movements and some movements to avoid seemed to be well understood by the participants; however, they often felt they lacked resources on accessing the professionals to provide that advice. Most participants seemed to clearly understand that seeking other health professionals for advice on exercise was for the therapeutic benefit of building strength and 
preventing falls, and likely would not contribute to rebuilding bone. There was, however, one optimistic participant that hoped exercise and nutrition modifications would rebuild bone.

"The logical part of my brain that what it's going to do is strengthen the muscles to support the bones so I'm more likely to be stable and prevent myself from falling or recover if I slip. The magical thinking is that, I did have a physiotherapist and a nutritionist say to me that um there is a possibility of increasing bone density with exercise."-Female, age 57

Although this participant could correctly identify that the exercise and nutrition would not help to rebuild bone, she was optimistically hoping that it might. All of the participants were able to correctly identify that falls prevention was necessary to prevent falls in an effort to reduce the risk of fractures.

\section{Lifestyle modifications}

\section{Modifying diet for osteoporosis}

When asked about modifying the diet for osteoporosis, all of the participants were knowledgeable about the importance of calcium consumption. Almost all of the participants were aware that calcium should be obtained through dietary sources and only if the dietary sources are insufficient (this was a concern for one of the participants who was vegan) were they to add supplements. All of the participants recognized that they should be taking Vitamin D supplements. However, there was confusion concerning why these supplements should be taken and how much. Few participants seemed to understand bioavailability of food sources, and they seemed to understand that sources like milk and yogurt were the best for calcium but were less aware that leafy greens also contain sources of calcium. Although few participants reported modifying their diet for their osteoporosis, there was a discussion on the fact that they already eat healthy and that should be sufficient for their osteoporosis as well. A couple of the participants sought advice from a dietitian to be able to meet their dietary goals and needs for osteoporosis, but most participants relied on the advice of their physicians for dietary advice.

\section{Balancing osteoporosis dietary advice with other dietary requirements}

Some participants talked about the challenge of managing osteoporosis dietary requirements while balancing the needs of other comorbid conditions that affected their dietary needs and restrictions. Although this was an important challenge, they felt that healthcare providers were often not prepared to balance different dietary indications and restrictions. Participants expressed their concerns that the specialists care for one disease, but rarely seem to provide advice that may suit all their needs. One participant illustrated this conflict when trying to optimize her diet for cancer and osteoporosis:

"after I had cancer I went to a naturopath, and there's a whole school of thinking that doesn't like calcium because it along with sugar and other thing's in your diet are inflammatory agents. And I know that person was giving me enormous calcium pills, but they were really giving me a recommendation around eliminating dairy. But of course, the information you get from the dietician and that would be very different. And I don't know if I have a completely comfortable resolution around those questions. Because I have always included a lot of dairy and avoided some of the anti-inflammatory diets that they will give to post-cancer people. Just because of my concern about bone density. And dairy is the fastest way to get $300 \mathrm{mg}$ of calcium per cup"-Female, age 61.

Page $12 / 21$ 
Sometimes the dietary restrictions were indicated by another comorbid health condition, such as obesity. However, attaining the recommended calcium intake for someone with osteoporosis was confounded by food intolerances or preferences. One participant overcame her need to avoid milk products because of stomach problems by substituting for other calcium rich products: "I'm very careful because I don't have dairy, I supplement with water buffalo yogurt, kiefer, which is really good, even better than yogurt, and goats' milk, and all those things. I have to work around my dietary issues"-Female, age 70.

Overall, participants recognized that diet was important, but they often felt that they had to explore solutions independently on the Internet since healthcare providers were not able or willing to provide customized dietary solutions for their individual needs. In doing so, they also expressed concern about whether the information they would glean this way would be valid.

\section{Reducing falls risk through home and environment modifications}

To reduce the risk of falls, several of the participants have made home or lifestyle modifications. Two of the oldest participants in the study continue to live independently and attribute their ability to maintain independence to home modifications to reduce the risk of falling:

"P1-I mean we have taken out carpets but that's not only for him... my son in law, came in and started to trip and then [my husband] came in behind him and did the same thing. So I thought that's it.

P2-yeah they're really hazardous.

P1- the only thing we've done is the shower for him. We put a chair in. But he doesn't need it anymore. It was for when his arm was broken. But we leave it there. And I find that I sit there when I'm drying my feet off now. I rather like it. But we talked about if and when we need a stair lift we can put that in." - P1: Female age 88, and P2: Male age 90 .

Other participants mentioned buying winter shoe grips to reduce the risk of falls, or ensuring they use a railing when walking up and down stairs.

\section{Learning to exercise independently for osteoporosis Exercise self-efficacy}

One of the key benefits of engaging in an exercise program for someone with osteoporosis was to learn about which exercises were safe and unsafe to do. Beyond learning what was safe, there was a sense of establishing osteoporosis exercise self-efficacy. One participant had already learned exercises for a comorbid condition but now felt she had to learn new ones specifically for her osteoporosis:

"Most of my exercises were geared towards my vestibular and building up muscle from my accident and now that I'm dealing with osteoporosis, that's a whole new set of exercises and strengthening. You're targeting specific so that will help overall. And I think it also helps you gain more flexibility and things to prevent falls." -Female, age 62.

As well, although some participants looked to online resources, like Osteoporosis Canada, for advice on how to manage their osteoporosis, but found they were not always relevant to their needs. The online resources did not 
seem to provide sufficient information for an already active individual to learn how to modify her exercise routine to be safe for osteoporosis. She said:

"the Osteoporosis Canada website didn't fit my profile. I read the article that was poste by one of the doctors there, but I found it very alarming. It was like don't do shoulder press, don't do jumping jack. It was very limiting in what you should be doing. Instead of encouraging you to be an active individual." --Female, age 51.

It seemed there was a need for personalized exercise prescription from a trusted and competent health care professional, with knowledge on how to modify exercises for people with osteoporosis based on their activity profile, experience with exercise, and risk of fracture.

\section{Learning types of exercise, proper form and exercise progressions}

Learning how to exercise for someone with osteoporosis went beyond identifying exercises and required an explanation of proper form and how to progress the exercise. One participant went to see a kinesiologist specializing in exercise for people with osteoporosis and said that the expectations were:

"that [the kinesiologist] would help me identify what was safe to do, that would not in the long run hurt me or jeopardize my body in any way that [the kinesiologist] would help me strengthen and build and get stronger. So that it's not status quo, and that [she's] continuing to help me progress." -- Female, age 51.

Finding a reliable exercise specialist was perceived as being valuable by all of the participants.

Non-Healthcare Providers

In addition to health professional advice, participants valued hearing about the personal experiences of others living with osteoporosis. Specifically, some of the participants sought advice from their relatives, spouses, or friends, some of whom had osteoporosis, but some of whom did not have osteoporosis.

\section{Corroborating health professional advice}

Although many of the participants agreed that seeing a health care professional is important, there seemed to be a sense of relying on non-healthcare provider advice to corroborate the advice they were given from their health care professional. For example, one participant's wife had osteoporosis and he said:

"Well the first time broke my arm, that's when I went to go see [the specialist]. So I hadn't had experience. My wife is also a patient of [the specialist] and has been for a long time.. So I was familiar with osteoporosis through that."-Male, age 90.

Beyond relying on his wife for help managing the pharmacological side of his osteoporosis, he also relied on her to facilitate the advice provided to him about his non-pharmacological needs. Throughout the interview he continued to mention that his wife would accommodate their meals to incorporate protein and calcium, which were recommended by their physician to better manage their osteoporosis. She would also facilitate making his physiotherapy appointments, to help recover after his wrist fracture and reduce his risk of falling.

\section{Validation of their belief system}


For participants that relied on non-healthcare provider advice, there was a sense of their belief system emerging as they were discussing the type of advice they sought and the type of advice they adhered to. One woman was a strong believer in alternative medicine, relying mostly on vitamins for her treatment of osteoporosis. When asked where she learned about managing her osteoporosis in this way she said:

"Oh just regular people that have family members or my friends that were doctors or my friends that were nurses, or friends that were nutritionists. All kinds of people. And some of them were professional people, some of them were not, just regular. And I found out and I think it was just the one time. And I think it was a man that told me this and I don't think he was a professional doctor or anything but he said you know my grandma has been taking what's been called collagen. And oh my god it really helps her with her joints and it helps her with this and that. And I thought hm interesting. Collagen. Collagen is something that our body produces to keep us young, maybe that is something I should take. And I researched and found something called Genacol, at that time"-Female, age 62

This woman did not feel that she was an exercise type of person and therefore tried to rely on other nonpharmacological methods for dealing with her osteoporosis. Another woman wanted to treat her osteoporosis more naturally and relied on the advice of the Internet to use Alfalfa roots to heal a fractured bone. Both women experienced perceived success with their alternative medicine strategies, suggesting that their belief system was validated.

\section{Learning from other's experience}

It was common for the participants in this study to receive advice from other people with osteoporosis, or other people that had experienced similar situations to them. The participants in this study felt that advice from others who experienced a similar situation was a way to learn about osteoporosis disease management. A lot of the participants relied on family members such as mothers and fathers who had gone through osteoporosis, to learn how to manage their own osteoporosis. When asking one woman about her knowledge related to falls prevention, she mentioned she was knowledgeable:

“...because of my mom. She's been to the falls prevention clinics over the years and obviously with [her husband] I knew right away and I was the one that said you have to have a walker now. And he didn't want it at first, but of course he didn't even want to have the cane. But now that he's using it, he's so on board. He sees how important it is and how much more comfortable he is. And so I think I have a good knowledge of the process. And I have a lot of aches and pains in my hips and my knees and so I tend to hold the railings all the time. I'm a total rail holder." Female, age 61

For this participant, she had experience with her mother and her father having osteoporosis, falling and having a fracture. She understood the importance of fall prevention. She applied these fall prevention strategies to both her home and her workplace. The workplace was a good place to implement falls prevention strategies because workplace safety was a priority, which translated to their home life. Other people use the advice of other's experience to try alternative medicine or seek advice from a personal trainer or physical therapist that was referred to them by a friend or family member. It did not emerge that people sought advice from others about going on medication but would seek advice if their medication was giving negative side effects and used that as validation to end medication use. 


\section{Discussion}

This study uniquely identified that people living with osteoporosis seek different types of information and have different knowledge access behaviours and experiences with physicians, other health professionals and nonhealthcare providers. Specifically, when interacting with physicians, patients with osteoporosis want to develop a therapeutic relationship that can help them understand osteoporosis as a disease, how it might progress, and the timing and role for non-pharmacological and pharmacological treatments. They also want more in depth discussion of the potential risks and benefits of different approaches to contribute to what are sometimes challenging choices. People living with osteoporosis engage in discussions with other health professionals for a more in-depth understanding of non-pharmacological care in osteoporosis management, help with the lifestyle modifications associated with managing osteoporosis and learning how to exercise safely and independently. People living with osteoporosis also value the experiences, opinions and advice from people who are not healthcare providers, particularly other people living with osteoporosis. In doing so they want to understand the experiences of what treatments are like from the user perspective, which is something that healthcare providers often cannot provide. In other cases, they wish to corroborate the advice from health care professionals as a way to cross-validate their beliefs, or potentially to determine how important certain treatment options are for other people in their lives. Although previous studies have identified that osteoporosis management requires the collaboration of many health professionals, this study helps to better understand the patient's perspective in navigating the osteoporosis health information within a health care system where multiple providers are likely to be involved in their management.

We found that patients rely on their physician to explain osteoporosis as a disease, and the role of pharmacology in managing their disease. This finding is consistent with other studies identifying that physicians play a large role in the disease management, especially from a pharmacological perspective[2, 9, 14]. Although some of the participants interviewed in this study accepted their diagnosis, there were others that expressed not taking it seriously due to not feeling the disease, not willing to accept the disease as it might mean modifying their lifestyle, or not fully understanding the severity of osteoporosis. This differs from other findings that say patients with osteoporosis had an "Aha" moment to understand their fracture risk[14], and accepting that their bones are frail[7]. This "Aha" moment likely came because all of the participants in the study by Hjalmarson et al., (2007) had experienced a fragility fracture, whereas the participants in the current study did not. People with osteoporosis have feelings of denial where they cannot believe that they have the disease[7, 8, 14], so explaining the treatment options seemed like one strategy to improve the navigation of disease management.

People with osteoporosis expressed a desire to be empowered to manage their osteoporosis. The participants had concerns about not having sufficient information to make informed decisions about their osteoporosis care, some felt that they either did not have knowledge on how best to manage their osteoporosis, or which healthcare professional to talk to, and most concerning for many of the participants seemed to be around not knowing which questions to ask. More effective education for people living with osteoporosis through better health resources and communication channels is likely to result in greater empowerment. Empowering patients has been proposed in other qualitative studies in people with osteoporosis[7, 15-17]. Previous studies suggest that patients need to be aware of the risks, balance their emotions, and to be physically and socially active with a high quality of life[7]. Health literacy may also contribute to feelings of empowerment[18]. If patients can be educated to improve their health literacy, it may help improve feelings of empowerment to manage their disease[18]. Given that participants expressed concerns about not knowing what to ask their health care professionals, we compiled a list of

Page 16/21 
questions that emerged from the interviews as questions participants might ask their health care professionals to facilitate their understanding of how to manage their osteoporosis (Table 2).

The results from this study can help health professionals to understand what areas they should uniquely focus on when helping patients manage their osteoporosis. For example, patients expressed that decisions around using medication were unique to physicians, and that often there was insufficient time to have a robust discussion of the pros and cons for timing of pharmacological options. More time and decision support tools might improve the communication and decision-making process. Therapeutic alliance is a complex and important part of use of health information for people living with osteoporosis. A qualitative study of participants with osteoporosis, found that participants who had the least contemplation and had the easiest time deciding to take osteoporosis medication were those that had trust in their healthcare provider[19]. To facilitate the dissemination of knowledge about the benefits of non-pharmacological strategies could be the use of educational resources. Educational resources have been used to facilitate communication between patients and physicians[20-22], and could also support the physicians in providing non-pharmacological information[9] and allowing time and discussion to contribute to shared decision-making where patients would have less decisional conflict and be more likely to adhere to the recommendations. However, given that proper exercise prescription and instruction requires a physical evaluation, evaluation of preferences, physical and health literacy and review of medical/osteoporosis history so that the clinician can properly target the exercise to the individual, it is unlikely that physicians will have the time to effectively take on this responsibility. In this scenario, physicians play an important role in educating patients about the importance of diet exercise and referring them to appropriate healthcare professionals who can provide detailed instruction and customized management plans. It was clear that patients thought about risks, benefits and how these evolve over time as important to making decisions about the timing or necessity of medications. Therefore, incorporating this longitudinal perspective in a medication that is meant to prevent future problems is an important aspect that is unique to medications that are more preventive than curative.

Other health professionals have a role of discussing non-pharmacological options with people with osteoporosis. Some topics might include the benefits and limitations of exercise, nutrition and falls prevention, when to seek physician advice, manage the lifestyle modifications such as reducing falls risk, modifying diet and encouraging an exercise routine that meets pre-osteoporosis goals. As well, teaching people with osteoporosis to exercise independently by increasing exercise self-efficacy and learning how to exercise safely with osteoporosis. It is important to recognize that non-healthcare providers contribute to the management of osteoporosis, and some of the erroneous beliefs may need to be discussed by the healthcare professionals, so people with osteoporosis are appropriately managing their disease. Dietary recommendations must consider that different patients will have comorbid health conditions, food allergies, food access/security issues, and cultural or religious beliefs that influence what foods they are willing and able to eat. Prior exercise behaviours, physical literacy, environmental context, personal preferences, seasonal weather, access to exercise equipment and other factors will also influence what exercise programs people are willing and able to execute and maintain. It was clear that personal and environmental factors play a large role in decision-making and behaviour maintenance for people living with osteoporosis. This may be one reason why participants find discussion with other people living with osteoporosis or within their social circles so valuable. Strategies to optimize adherence to osteoporosis treatment must consider these personal and environmental factors. 
The strength of this study is that we were able to identify key knowledge providers used to disseminate knowledge on osteoporosis management to people with osteoporosis. A variety of patients with osteoporosis were interviewed with an age range of 51-90, including men and women, from a variety of ethnical backgrounds and from both rural and urban cities. Through these interviews we were able to receive in depth knowledge on how people with osteoporosis receive information about their osteoporosis management. This study methodology presents some limitations. Firstly, it is acknowledged by the author that there may be personal bias associated with the analysis and questions, the methodology was selected to account for that and a personal reflection was added in Appendix 2. The participants were eager to participate in the study and may have been more proactive with their health care professionals to seek information, potentially leaving themes unknown for patients that are very passive with their osteoporosis care. As well, all participants were recruited from one clinic, potentially limiting the diversity of the population. Future studies should confirm these results and evaluate the perspective from more males. It was not clear the timeline from when patients last saw their physician, or healthcare providers which may add some recall bias.

\section{Conclusion}

In conclusion, this study identified information-seeking experiences for people with osteoporosis, about managing their osteoporosis. We found that people with osteoporosis rely on knowledge from their physician, other health professionals and non-healthcare providers, but ultimately would wish to feel empowered with knowledge to manage their osteoporosis and know which questions to ask. Future studies should confirm the themes that emerged from this study and may assess the extent to which this information can be used to improve communication and information access for people living with osteoporosis, or guide future resource development to address patient specific information needs

\section{Abbreviations}

BMD

Bone Mineral Density

\section{Declarations}

Ethics approval and Consent to participate All procedures performed in studies involving human participants were in accordance with the ethical standards of the institutional and/or national research committee. Informed consent was obtained from all individual participants included in the study

Consent for publication All participants provided written consent for their data to be published

Availability of supporting data The datasets used and/or analysed during the current study available from the corresponding author on reasonable request

Conflicts of interest/Competing interests CZ, JM, RF, MS, NS, and AK report no conflicts of interest

Funding Authors received no specific funding for this work.

\section{Authors' contributions}

Page $18 / 21$ 
$\mathrm{CZ}$, came up with the idea, collected the data, analyzed the data, wrote the manuscript and applied edits. CZ read and approved the final version of the manuscript and agreed to be personally accountable for their own contribution to the manuscript

$\mathrm{JM}$, came up with the idea, provided resources and supervision, and provided edits. JM read and approved the final version of the manuscript and agreed to be personally accountable for their own contribution to the manuscript

$\mathrm{RF}$, analyzed the data, and provided edits. RF read and approved the final version of the manuscript

MS, provided resources and supervision, and provided edits. MS read and approved the final version of the manuscript and agreed to be personally accountable for their own contribution to the manuscript

NS, provided resources and supervision, and provided edits. NS read and approved the final version of the manuscript and agreed to be personally accountable for their own contribution to the manuscript

AK provided resources and supervision, and provided edits. AK read and approved the final version of the manuscript and agreed to be personally accountable for their own contribution to the manuscript.

\section{Acknowledgements:}

Christina Ziebart is supported by the CIHR Doctoral Award. Joy C MacDermid was supported by a CIHR Chair in Gender, Work and Health and the Dr. James Roth Research Chair in Musculoskeletal Measurement and Knowledge Translation

\section{References}

1. Burge R, Dawson-Hughes $\mathrm{B}$, Solomon $\mathrm{DH}$, et al. Incidence and economic burden of osteoporosis-related fractures in the United States, 2005-2025. Journal of bone mineral research. 2007;22(3):465-75.

2. Papaioannou A, Morin S, Cheung AM, et al. 2010 clinical practice guidelines for the diagnosis and management of osteoporosis in Canada: summary. Cmaj. 2010;182(17):1864-73.

3. Giangregorio L, Papaioannou A, Macintyre N, et al. Too fit to fracture: exercise recommendations for individuals with osteoporosis or osteoporotic vertebral fracture. Osteoporos Int. 2014;25(3):821-35.

4. Khan A, Fortier M, Reid R, et al. Osteoporosis in menopause. Journal of Obstetrics Gynaecology Canada. 2014;36(9):839-40.

5. Cortet B, Bénichou O. Adherence, persistence, concordance: do we provide optimal management to our patients with osteoporosis? Joint Bone Spine. 2006;73(5):e1-7.

6. Rossini M, Bianchi G, Di Munno O, et al. Determinants of adherence to osteoporosis treatment in clinical practice. Osteoporos Int. 2006;17(6):914-21.

7. Hjalmarson HV, Strandmark M, Klässbo M. Healthy risk awareness motivates fracture prevention behaviour: A grounded theory study of women with osteoporosis. International Journal of Qualitative Studies on Health Well-being. 2007;2(4):236-45.

8. Ziebart C, McArthur C, Lee L, et al. "Left to my own devices, I don't know": using theory and patient-reported barriers to move from physical activity recommendations to practice. Osteoporos Int. 2018;29(5):1081-91. 
9. Clark R, McArthur C, Papaioannou A, et al. "I do not have time. Is there a handout I can use?": combining physicians' needs and behavior change theory to put physical activity evidence into practice. Osteoporos Int. 2017;28(6):1953-63.

10. McArthur C, Ziebart C, Papaioannou A, et al. "We get them up, moving, and out the door. How do we get them to do what is recommended?" Using behaviour change theory to put exercise evidence into action for rehabilitation professionals. Archives of osteoporosis. 2018;13(1):7.

11. Strauss A, Corbin J. Grounded theory methodology. Handbook of qualitative research. 1994;17(1):273-85.

12. Hunt MR. Strengths and challenges in the use of interpretive description: reflections arising from a study of the moral experience of health professionals in humanitarian work. Qual Health Res. 2009;19(9):1284-92.

13. Thorne S, Kirkham SR, O'Flynn-Magee K. The analytic challenge in interpretive description. International journal of qualitative methods. 2004;3(1):1-11.

14. Beaton DE, Sujic R, Mcllroy Beaton K, et al. Patient perceptions of the path to osteoporosis care following a fragility fracture. Qual Health Res. 2012;22(12):1647-58.

15. Zimmerman MA, Rappaport J. Citizen participation, perceived control, and psychological empowerment. American Journal of community psychology. 1988;16(5):725-50.

16. Zimmerman MA, Warschausky S. Empowerment theory for rehabilitation research: Conceptual and methodological issues. Rehabilitation psychology. 1998;43(1):3.

17. Laverack G. Health promotion practice: power and empowerment. Sage; 2004.

18. Almond H, Cummings E, Turner P. Empowerment. Digital Literacy and Shared Digital Health Records: the value of 'nothing about me without me'. Studies in Health Technology Informatics. 2019;266:13-9.

19. Sale JE, Gignac MA, Hawker G, et al. Decision to take osteoporosis medication in patients who have had a fracture and are'high'risk for future fracture: A qualitative study. BMC Musculoskelet Disord. 2011;12(1):92.

20. Khan AA, Kaiser S. Atypical femoral fracture. CMAJ. 2017;189(14):E542-2.

21. Khan AA, Morrison A, Hanley DA, et al. Diagnosis and management of osteonecrosis of the jaw: a systematic review and international consensus. J Bone Miner Res. 2015;30(1):3-23.

22. Khan AA, Morrison A, Kendler DL, et al. Case-based review of osteonecrosis of the jaw (ONJ) and application of the international recommendations for management from the international task force on ONJ. Journal of clinical densitometry. 2017;20(1):8-24.

\section{Figures}




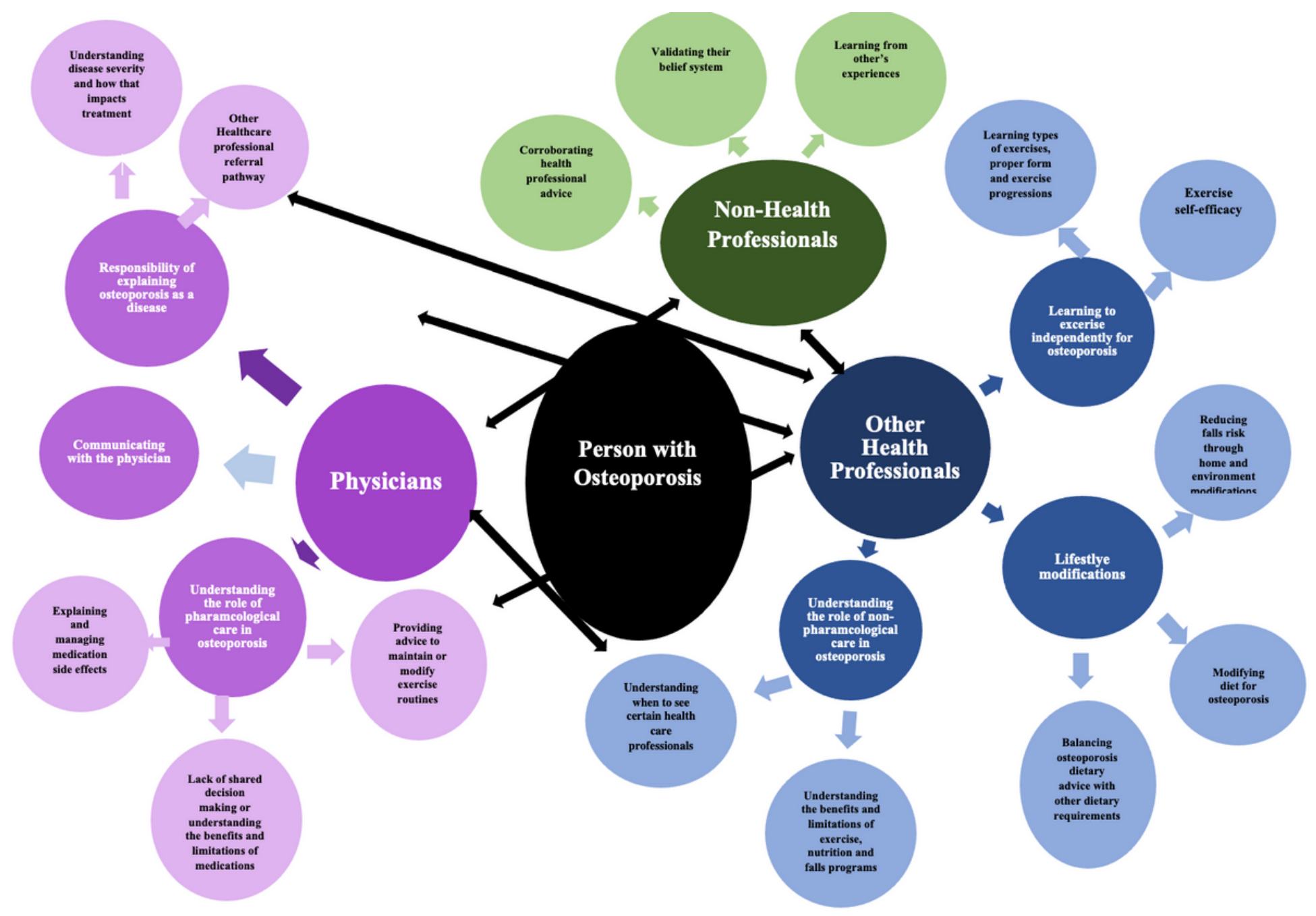

\section{Figure 1}

Model of themes that emerged from the interviews. The person with osteoporosis remains at the center and is responsible for navigating their information gathering through their management of osteoporosis. The other knowledge stakeholders are the physician, other health professionals and non-health professionals.

\section{Supplementary Files}

This is a list of supplementary files associated with this preprint. Click to download.

- Appendix1.docx

- AuthorReflectionAppendix2.docx 